\title{
Timpanoplastía: Revisión y experiencia de 4 años en el Hospital Clínico de la Universidad Católica de Chile
}

\author{
Tympanoplasty: Review and experience of 4 years at the \\ Hospital Clínico de la Universidad Católica de Chile
}

Raúl Garfias $\mathrm{L}^{1}$, Tomás Andrade $\mathrm{D}^{2}$, Ximena Maul F², María de los Ángeles Baeza $\mathrm{A}^{1}$, Jorge Caro $\mathrm{L}^{\mathbf{1}}$.

\section{RESUMEN}

Introducción: Desde la introducción de esta técnica quirúrgica en la segunda mitad del siglo XIX ha habido múltiples publicaciones sobre diversas experiencias y nuevas técnicas a nivel nacional e internacional. En nuestro hospital esta cirugía es parte de la práctica clínica habitual.

Objetivo: El objetivo general es revisar la experiencia en timpanoplastías en el Hospital Clínico de la Universidad Católica de Chile en los últimos 4 años. Los objetivos específicos son revisar la epidemiología de los pacientes intervenidos, las técnicas quirúrgicas utilizadas y los resultados anatómicos y audiológicos conseguidos.

Material y método: Estudio retrospectivo y descriptivo. Se revisó la totalidad de las fichas y audiometrías de los pacientes sometidos a timpanoplastías que cumplieron criterios de inclusión y exclusión bien definidos. Se incluyeron sólo pacientes cuya cirugía fue realizada entre enero de 2007 y diciembre de 2010.

Resultados: Se obtuvo éxito anatómico en $82,4 \%$ de nuestros pacientes, con una tasa de complicaciones de $17,57 \%$. Con respecto a los resultados auditivos, se observó un éxito auditivo total de 87,08\%, y sólo en 13\% de los pacientes empeoró su audición.

Conclusiones: Los resultados anatómicos y auditivos obtenidos son comparables a lo publicado en la literatura nacional en la última década. Nuestra experiencia muestra un buen rendimiento con las técnicas e injertos utilizados, así como un buen resultado en las cirugías realizadas por médicos residentes, similares a los obtenidos por médicos staff.

Palabras clave: Timpanoplastía, miringoplastía, otitis media crónica, otopatía adhesiva.

\begin{abstract}
Introduction: Since the introduction of this surgical technique in the second half of the nineteenth century, there have been many national and international publications on various experiences and new techniques. In our hospital this surgery is part of the routine clinical practice, so we decided to conduct this study to review our experience over the last 4 years.

Aim: The general objective is to review the experience of tympanoplasty in the Hospital Clínico de la Universidad Católica de Chile in the last 4 years. The specific objectives are to review the epidemiology of patients undergoing this surgery, the surgical techniques used and the anatomical and audiological results achieved.
\end{abstract}

\footnotetext{
'Médico del Departamento de Otorrinolaringología, Hospital Clínico de la Pontificia Universidad Católica de Chile.

${ }^{2}$ Médico Cirujano, Pontificia Universidad Católica de Chile.
} 
Material and method: Descriptive and retrospective study. We reviewed all the clinical records and hearing tests in patients undergoing tympanplasty who met inclusion and exclusion criteria clearly defined. We only included patients whose surgery was performed between January 2007 and December 2010.

Results: Anatomical success was archieved in $82.4 \%$ of our patients, with a complication rate of $17.57 \%$. With regard to hearing results, there was a total audiological success of $87.08 \%$ and only $13 \%$ of our patients experienced worsening of their audition.

Conclusions: The anatomical and audiological results obtained are comparable to those published in the national literature in the last decade. Our experience shows a good performance with the techniques and grafts used, and good results in surgeries performed by residents, similar to those obtained by staffs.

Key words: Tympanoplasty, miringoplasty, chronic otitis media, adhesive otitis media.

\section{INTRODUCCIÓN}

La timpanoplastía es una cirugía de reconstrucción del mecanismo de conducción del oído medio, con o sin la utilización de injertos. No sólo incluye la reparación de la membrana timpánica, sino la totalidad de técnicas que permiten restaurar el funcionamiento de este sistema ${ }^{1}$.

La primera timpanoplastía reportada fue realizada por Berthold en 1878, pero no fue hasta 1952 en que se plantearon los conceptos quirúrgicos actuales de esta cirugía, presentados por Wullstein y Zöllner. Desde 1953 House ya apoyaba el uso de piel libre como injerto para la reparación de la membrana timpánica. Posteriormente, Wullstein clasificó las timpanoplastías en 5 tipos, clasificación bastante conocida y utilizada hasta nuestros días².

Por otro lado, en Chile se comenzó a publicar resultados de esta cirugía a mediados de la década de los cincuenta. Inicialmente, autores como Maspetiol, Barroilhet, Emhart y Otte publicaron sus primeras experiencias ${ }^{3-6}$. La técnica fue evolucionando, y se publicaron éxitos anatómicos hasta $72 \%$ en la década de los ochenta, destacando los trabajos de Darritchon, Caro y Plaza ${ }^{7-9}$. En Ios últimos años, y en particular en la última década, se han publicado múltiples experiencias en distintos centros, alcanzando éxitos anatómicos hasta $90 \%$ y éxitos auditivos hasta $92 \%{ }^{10-14}$.

\section{OBJETIVO}

El objetivo general de este estudio es revisar la experiencia de los últimos 4 años en timpanoplastías realizadas en el Hospital Clínico de la Pontificia Universidad Católica de Chile. Los objetivos específicos son realizar una descripción epidemiológica de los pacientes intervenidos, las técnicas quirúrgicas y sus variantes utilizadas y los resultados anatómicos y auditivos conseguidos.

\section{MATERIAL Y MÉTODO}

Se realizó un estudio retrospectivo y descriptivo en que se revisaron la totalidad de fichas de los pacientes sometidos a timpanoplastía en nuestro centro, entre enero de 2007 hasta diciembre de 2010.

\section{Criterios de inclusión:}

- Diagnóstico de otitis media crónica no colesteatomatosa

- Otopatía fibroadhesiva

- Ficha clínica y protocolo operatorio con información clara y suficiente

- Audiometría pre y posoperatoria

- Seguimiento de al menos 6 meses poscirugía

\section{Criterios de exclusión:}

- Perforación timpánica traumática

- Perforación timpánica poscirugía (postubo de ventilación)

- Cirugía de reconstrucción funcional poscirugía radical de oído

De los pacientes seleccionados, se rescataron datos clínicos y quirúrgicos desde su ficha y 
protocolo operatorio, y se revisaron los registros de audiometrías presentes en nuestro centro.

Se registraron los siguientes datos:

- Datos epidemiológicos: Edad, sexo, antecedentes mórbidos, diagnóstico preoperatorio, oído operado, estado de oído contralateral y antecedente de timpanoplastía previa en el oído operado.

- Datos de la cirugía: Vía de abordaje, tipo de injerto, técnica quirúrgica, tipo de timpanoplastía (según la clasificación clásica o de Wullstein), uso de injertos de cadena osicular y primer cirujano (staff 0 residente).

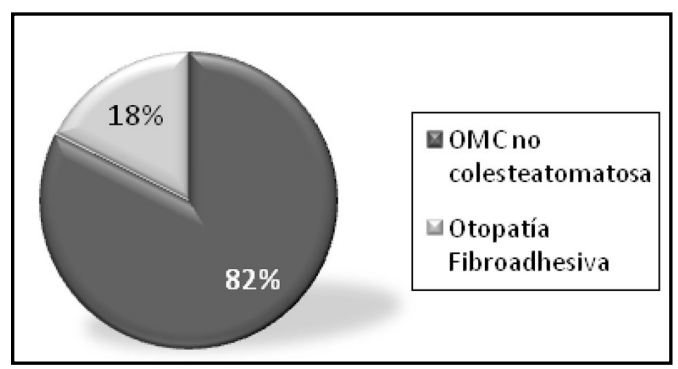

Figura 1. Diagnóstico preoperatorio.

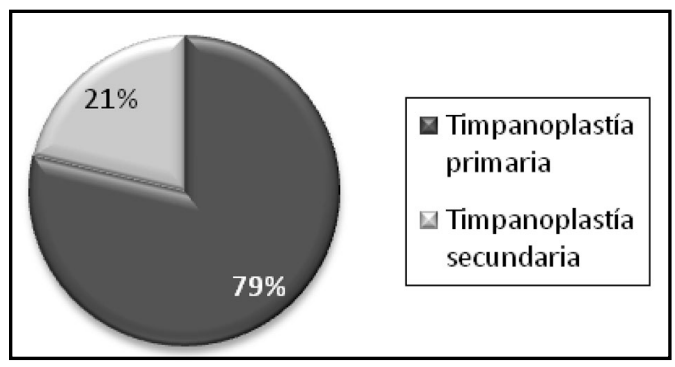

Figura 2. Antecedente de timpanoplastía previa.

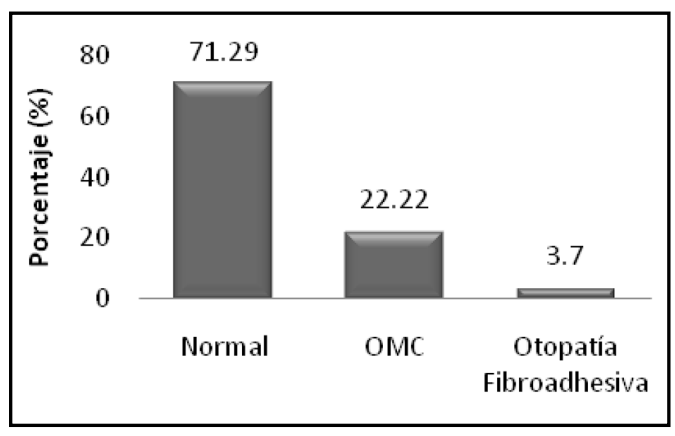

Figura 3. Estado de oído no intervenido.
- Datos de seguimiento: Meses de seguimiento, resultado anatómico y promedios tonales puros (PTP) a los 500, 1.000 y $2.000 \mathrm{~Hz}$ en audiometrías pre y posoperatorias.

Para analizar el éxito auditivo, se clasificó a los pacientes en 3 grupos: Buen resultado (mejoría en PTP aéreo mayor 0 igual a $10 \mathrm{~dB}$ con respecto al preoperatorio), resultado satisfactorio (mejoría en PTP aéreo entre 0 y $10 \mathrm{~dB}$ con respecto al preoperatorio) y mal resultado (empeoramiento del PTP aéreo con respecto al preoperatorio). Se consideró éxito auditivo total como la suma aritmética entre los 2 primeros grupos.

\section{RESULTADOS}

En el período de tiempo estudiado se realizaron 108 timpanoplastías que cumplieron con los criterios de inclusión y exclusión.

La mayoría de los pacientes $(64,81 \%)$ correspondía a personas en edades medias de la vida, entre 20 y 64 años. La media aritmética fue de 33,92 años con una desviación estándar de \pm 18,09. La distribución por sexo fue equitativa, con un leve predominio del sexo femenino (56\%).

La gran mayoría de los pacientes intervenidos no tenían antecedentes mórbidos de importancia $(82,4 \%)$. En los pacientes portadores de estos antecedentes, predominaban enfermedades prevalentes en la población general (hipertensión arterial, diabetes mellitus, hipotiroidismo, etc.).

El diagnóstico preoperatorio más frecuente fue la OMC no colesteatomatosa ( $82 \%$ ), seguida por la otopatía fibroadhesiva (18\%) (Figura 1). De la muestra total, $60 \%$ correspondió a cirugías en oído derecho, siendo timpanoplastías primarias en el $79 \%$ del total de los casos (Figura 2).

El 22,22\% de los pacientes eran portadores de una OMC no colesteatomatosa en el oído contralateral, y sólo el 3,7\% de otopatía fibroadhesiva (Figura 3).

Con respecto a la técnica quirúrgica, en la amplia mayoría (82\%) se utilizó técnica Austin. En menor proporción se utilizó técnica en Empalizada $(9 \%)$, House $(6 \%)$ y en Mariposa $(3 \%)$.

El abordaje fue mayoritariamente por vía endoaural (84\%) (Figura 4), utilizando en la mayoría de los casos injerto de cartílago-pericondrio 
para la reparación de la membrana timpánica $(77,77 \%)$ (Figura 5).

En relación al tipo de timpanoplastía, la mayoría de los pacientes fueron sometidos a una timpanoplastía tipo I (70,37\%). En menor proporción, timpanoplastías tipo IIIA $(24,07 \%)$ y IIIB $(5,55 \%)$. No se registraron pacientes sometidos a timpanoplastías tipo II, IV o V (Figura 6).

En las timpanoplastías tipo IIIA y IIIB se utilizaron injertos para la reparación de la cadena osicular. En las primeras se utilizó ampliamente autoinjertos de cartílago (84,61\%), y en menor proporción prótesis de titanio $(15,38 \%)$. En las segundas, se utilizó autoinjerto de cartílago en $66,66 \%$, y prótesis de titanio en $33,33 \%$. El número de ambas muestras fue de 26 y 6 pacientes, respectivamente (Figura 7).

Con respecto a los meses de seguimiento, la media fue de 15,55 meses con una desviación estándar de $\pm 12,15$. La mayoría alcanzó un seguimiento de 6 a 11 meses (56,48\%). Destaca que el

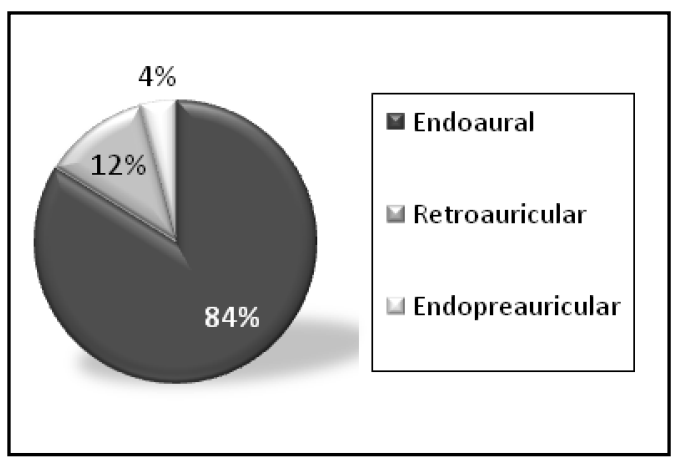

Figura 4. Vía de abordaje.

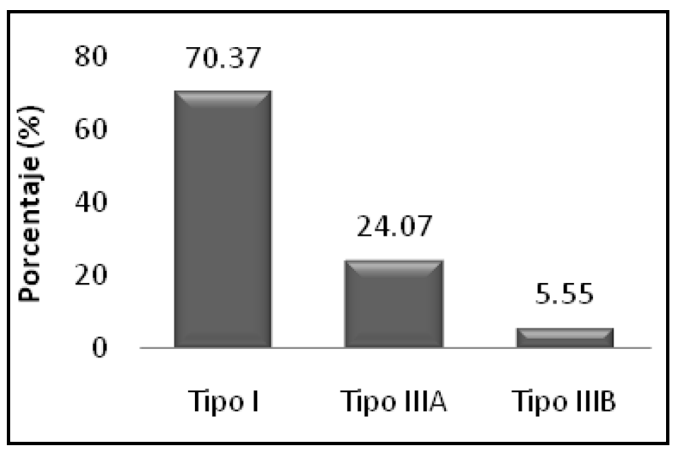

Figura 6. Tipo de timpanoplastía (clasificación de Wullstein).
$29,62 \%$ de los pacientes logró un seguimiento de 2 años 0 más (Figura 8).

La gran mayoría de los pacientes intervenidos presentaron un neotímpano indemne durante su seguimiento $(82,4 \%)$, observándose una tasa de complicaciones de 17,57\%. Las complicaciones registradas fueron: perforación del neotímpano $(12,96 \%)$, lateralización del injerto $(2,77 \%)$, desplazamiento de la prótesis $(0,92 \%)$ y granuloma posoperatorio $(0,92 \%)$. No se registraron casos de paresia facial (Figura 9).

Según vía de abordaje, el análisis muestra un éxito anatómico (neotímpano indemne durante el seguimiento) de $81,31 \%$ para la vía endoaural, $100 \%$ para la vía retroauricular y $100 \%$ para la vía endopreauricular.

Según el injerto utilizado, se observó un éxito anatómico de 79,26\% cuando se usó cartílagopericondrio. Por otro lado, el éxito anatómico obtenido con injerto de pericondrio y fascia tempo-

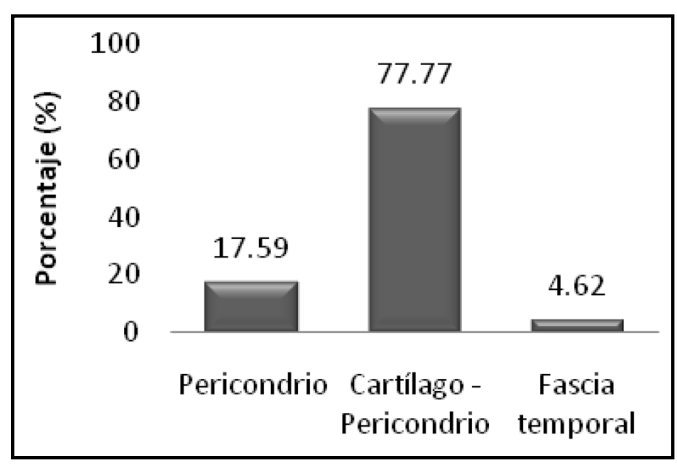

Figura 5. Injerto para reparación de membrana timpánica.

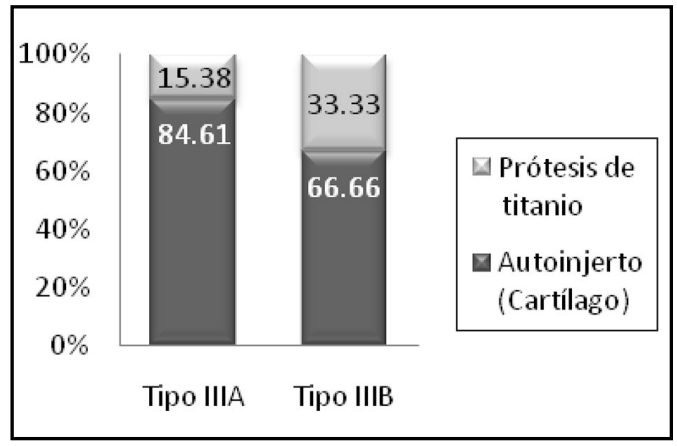

Figura 7. Injerto de cadena osicular. 


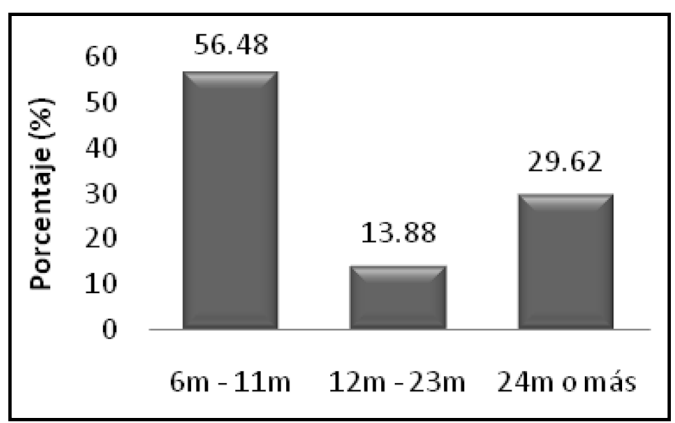

Figura 8. Meses de seguimiento posoperatorio.

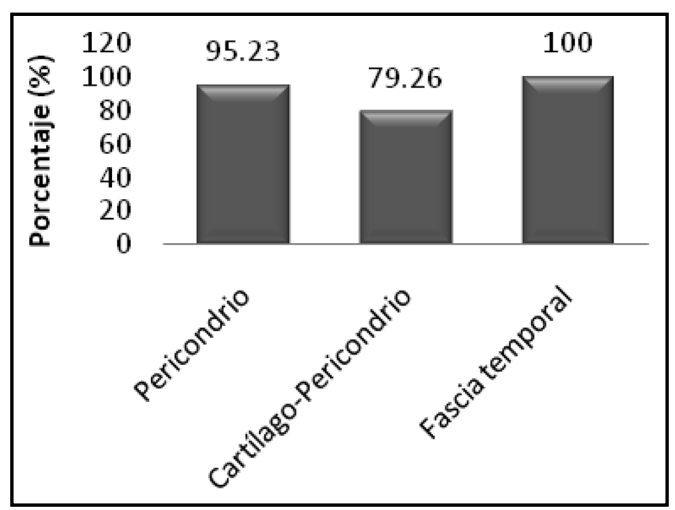

Figura 10. Éxito anatómico según tipo de injerto.

ral fue bastante elevado $(95,23 \%$ y $100 \%$, respectivamente) (Figura 10).

En relación a la técnica quirúrgica, en los pacientes intervenidos con técnica Austin se registró un éxito anatómico de $80,89 \%$. Las otras técnicas mostraron también éxitos anatómicos elevados, por sobre el $80 \%$ (Figura 11).

De acuerdo a la clasificación de éxito auditivo planteada anteriormente, $48 \%$ de los pacientes logró buenos resultados, 39\% logró resultados satisfactorios, y sólo 13\% presentó malos resultados. De lo anterior se obtiene un éxito auditivo total de $87,08 \%$ (Figura 12).

Dentro del análisis de éxito auditivo según tipo de timpanoplastía, destacan los peores resultados obtenidos en timpanoplastías tipo IIIA y IIIB al compararlas con los obtenidos en las timpanoplastías tipo I.

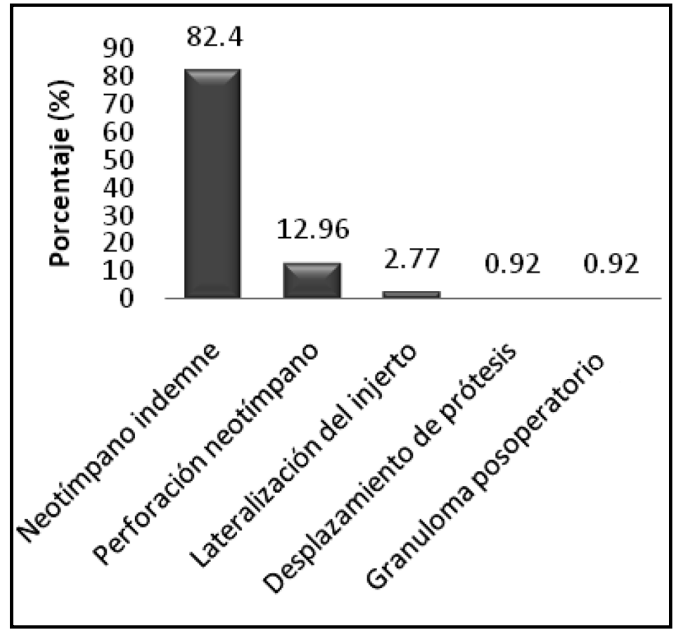

Figura 9. Resultado anatómico.

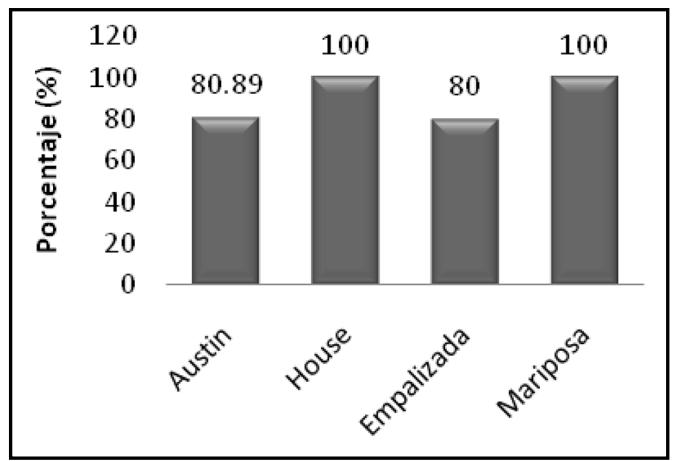

Figura 11. Éxito anatómico según técnica quirúrgica.

Los peores resultados fueron obtenidos en las timpanoplastías tipo IIIB, alcanzando el 33,33\% de malos resultados en este grupo (Figura 13).

Las cirugías realizadas por staff alcanzaron un éxito auditivo de $89,73 \%$. Por otro lado, las cirugías realizadas por residentes bajo supervisión alcanzaron un éxito auditivo de $86,94 \%$. Entre ambos grupos sólo se observa una variación entre la relación de buenos resultados versus resultados satisfactorios (mayor proporción de buenos resultados en el grupo de staff, versus mayor proporción de resultados satisfactorios en el grupo de residentes) (Figura 14). 


\section{DISCUSIÓN}

Del análisis de los datos obtenidos en nuestro estudio, podemos decir que la epidemiología de nuestros pacientes intervenidos es similar a la observada en otras publicaciones nacionales. Destaca que fueron operados mayoritariamente pacientes en edades medias de la vida, sin antecedentes mórbidos, con leve predominio del sexo femenino. Se observó un porcentaje no despreciable de patología de oído medio avanzada en el oído no intervenido, destacando la OMC no colesteatomatosa $(22,22 \%)$. Esto último muestra la importancia de mantener en control al paciente y favorecer el seguimiento, no sólo por la cirugía realizada, sino para pesquisar posible patología susceptible de tratamiento en el oído considerado sano.

Podemos observar que el diagnóstico preoperatorio más frecuente fue la OMC no colesteatomatosa, seguida por la otopatía fibroadhesiva. Se observa además un leve predominio de cirugías en el oído derecho $(60 \%)$, sin poder deducirse una causa clara para ello.

En relación a la técnica quirúrgica, vemos que en la mayoría de nuestros pacientes se utilizó la vía endoaural $(84 \%)$, con técnica Austin $(82 \%)$, y utilizando cartílago-pericondrio como injerto para la reparación de la membrana timpánica $(77,77 \%)$. Es importante mencionar que inicialmente en nuestro centro se utilizaba más la vía retroauricular (por su mayor exposición), usándose mayoritariamente injertos de pericondrio. Sin embargo, en la medida que se fueron abordando casos más complejos, con perforaciones de mayor tamaño o de mayor recurrencia, se comenzó a utilizar preferentemente injertos de cartílago o cartílago-pericondrio, dada su mayor estabilidad y rigidez. Esto último es respaldado por los estudios de Bernal y cols. y Stott y cols., que muestran éxitos anatómicos superiores con injertos de cartílago ${ }^{15-16}$. Debido a la gran experiencia que se ha logrado en nuestro hospital con dichos injertos, es que se han mantenido como los de elección para la reparación de la membrana timpánica tanto en casos de OMC no colesteatomatosa como en otopatía fibroadhesiva. Recalcamos que esto último, así como la preferencia por la vía endoaural y por la técnica Austin realizada a través de la perforación, son alternativas válidas para estos casos, siempre teniendo en

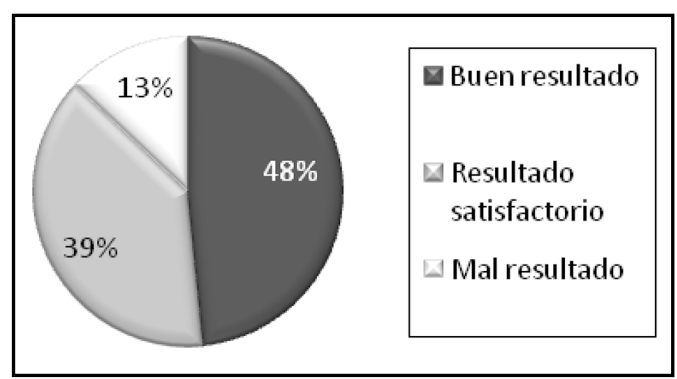

Figura 12. Resultado auditivo total.

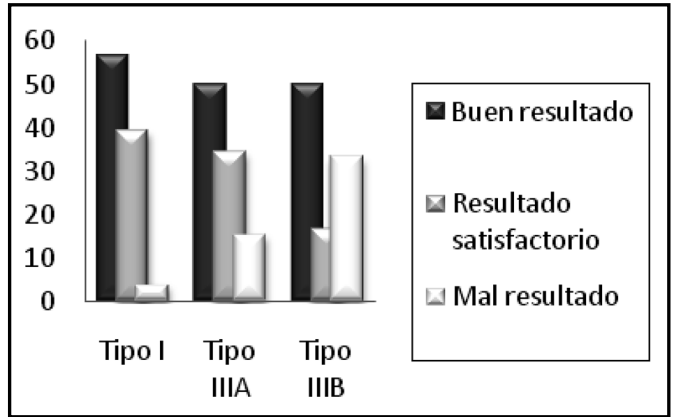

Figura 13. Resultado auditivo según tipo de timpanoplastía.

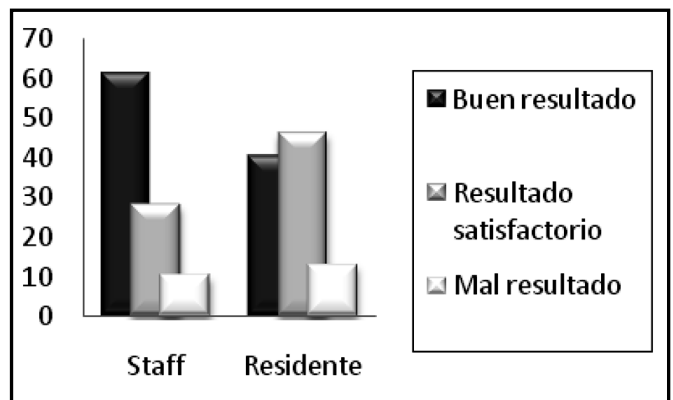

Figura 14. Resultado auditivo según cirujano.

cuenta la experiencia del cirujano o la experiencia local.

En nuestro estudio, se obtuvo en éxito anatómico de $82,4 \%$ y un éxito auditivo de $87,08 \%$. Ambos resultados son comparables a lo publicado en la última década en nuestro país ${ }^{10-14}$.

También es importante destacar que en nuestro estudio la gran mayoría de los pacientes se 
concentra en técnicas, vías de abordaje e injertos bien definidos (Austin, endoaural y cartílagopericondrio, respectivamente). Los otros grupos de pacientes muestran igualmente éxitos anatómicos y auditivos elevados, pero probablemente deban ser interpretados con cautela debido a su número más reducido.

Con respecto al tipo de timpanoplastía, destaca un mayor porcentaje de malos resultados al aumentar la complejidad quirúrgica, observándose un peor resultado auditivo en las timpanoplastías tipo IIIB al compararlas con las timpanoplastías tipo IIIA. Esto es similar a lo publicado por Nazar y cols, estudio en que se observó la misma tendencia, aunque sin existir diferencia significativa entre ambos grupos ${ }^{17}$. Las timpanoplastías tipo I muestran mejores resultados que los 2 tipos anteriores.

Finalmente, nuestro estudio revela resultados similares a nivel auditivo entre las cirugías realizadas por médicos staff versus residentes supervisados. El éxito auditivo total en ambos grupos fue similar (89,73\% versus $86,94 \%$, respectivamente), lo que permite concluir que, pese a tratarse de cirugías que requieren entrenamiento y supervisión inicial, pueden ser realizadas por médicos residentes en un programa de especialidad, sin que ello signifique una disminución importante en la calidad de los resultados.

\section{CONCLUSIONES}

El objetivo general de este estudio se encuentra ampliamente cumplido al revisar la experiencia en timpanoplastías realizadas en el Hospital Clínico. Los objetivos específicos, del mismo modo, se expresan en los resultados y discusión

La timpanoplastía es una cirugía frecuente en la práctica clínica otorrinolaringológica. La experiencia lograda en nuestro centro muestra resultados comparables a lo publicado en la literatura nacional, con tasas de éxito anatómico y auditivo satisfactorias. Creemos firmemente que el compartir la experiencia local con la comunidad científica de nuestro país es una de las bases para permitir el avance en la técnica quirúrgica y, así, mejorar los resultados en nuestros pacientes.

\section{BIBLIOGRAFÍA}

1. FIScH U. Tympanoplasty, mastoidectomy and stapes surgery. $1^{\text {st }}$ ed. Thieme: Stuttgart; 1994. p.2-40.

2. Albert Mudry, mD, PHD, Lausanne, Switzerland. History of myringoplasty and tympanoplasty type I. Otolaryngology Head and Neck Surgery 2008; 139: 613-14.

3. Maspetiol R. Indicaciones y técnica de la timpanoplastía (RR). Rev Otorrinolaringol 1956; 16: 17.

4. BARROILHET J. Nuestra experiencia en microcirugía del oído medio. Rev Otorrinolaringol 1958; 27: 38.

5. Emhart 0. Contribución al estudio de las timpanoplastías. Rev Otorrinolaringol 1959; 19: 109.

6. Otte Gabler J, Emhart 0, Cohn M. Timpanoplastías. Otitis media crónica supurada y su tratamiento quirúrgico. Rev Otorrinolaringol 1959; 19: 20.

7. Darritchon E. Evaluación de Timpanoplastías. Rev Otorrinolaringol 1978; 38: 72-4.

8. Plaza E. Análisis crítico de las timpanoplastías. Rev Otorrinolaringol Cir Cab Cue 1980; 40: 11-6.

9. Caro J y cols. Análisis de 168 timpanoplastías. Rev Otorrinolaringol Cir Cab Cu 1983; 40: 1723.

10. V Vergara T, M Arancibia S, J Maass 0 , L Tapia F, M Cumsille. Timpanoplastías: Revisión de 4 años. Rev Otorrinolaringol Cir Cab Cu 2001; 61: 102-6.

11. MP Vallejos, L Dentone. Timpanoplastías en mayores de 50 años. Rev Otorrinolaringol Cir Cab Cu 2003; 63: 100-5.

12. U Zelada, R Arregui, E Palacios. Timpanoplastía en niños: Experiencia de 10 años. Rev Otorrinolaringol Cir Cab Cu 2005; 65: 92-6.

13. C Toro, A Naser, C Sanhueza, C Valdés, JP Gormaz, V Molina, A LesLie. Timpanoplastías en adultos en el Hospital Clínico de la Universidad de Chile: Revisión de 10 años. Rev Otorrinolaringol Cir Cab Cu 2007; 67: 237-43.

14. De la Paz P, Pardo J, Fernández G. Timpanoplastías en adultos en el Complejo Asistencial Dr. Sótero del Río (CASR): Revisión 
de 5 años. Rev Otorrinolaringol Cir $\mathrm{Cab} \mathrm{Cu}$ 2009; 69: 221-226.

15. Bernal S, Tomas B. Indications, techniques and anatomic results of the tympanoplasty using palisade cartilage. Acta Otorrinolaringol Esp 1997; 48: 279-86.
16. Stott C, Inzunza P, Walker J, Bahamonde S. Timpanoplastía en empalizada. Rev Otorrinolaringol Cir Cab Cu 2002; 62: 238-242.

17. Nazar M, Bustos F, Larraguibel $H$, Larach J, IÑIGUEZ S. Timpanoplastías Tipo III. Rev Otorrinolaringol Cir Cab Cu 2003; 63:157-166.

Dirección: Dr. Raúl Garfias L.

Departamento de Otorrinolaringología, Hospital Clínico de la Universidad Católica de Chile Marcoleta $350,2^{\circ}$ piso

E mail: raulgarfias@gmail.com 